\title{
The Role of Interactions between Halloysite Nanotubes and 2,2'-(1,2-Ethenediyldi-4,1-phenylene) Bisbenzoxazole in Halloysite Reinforced Polypropylene Composites
}

\author{
By Mingxian LIU, Baochun GUO, ${ }^{*}$ Mingliang DU, and Demin JIA
}

The electron transferring interactions between halloysite nanotubes (HNTs) and 2,2'-(1,2-ethenediyldi-4,1-phenylene) bisbenzoxazole (EPB) was utilized to fabricate polypropylene (PP)/HNTs composite. Large HNTs aggregates are formed within the composites in the presence of EPB. The composites with EPB show substantially increased strength and modulus. The reinforcement mechanism of EPB incorporated PP/HNTs composites are elucidated to the combination of the stress transferring by the well dispersed HNTs and the energy dissipation by cracking the HNTs aggregates during the fracture process.

KEY WORDS: Halloysite / Electron Transferring / Polymer Composite / Reinforcement Mechanism /

Nanoparticles incorporated polymer composites was extensively investigated during the past two decades for their much higher performance compared with their microcomposites counterparts. ${ }^{1}$ Nanoclays constitute a class of nanoparticles widely used for the fabrication of nanocomposites. ${ }^{2}$ Due to the great discrepancy in polarity, the interfacial interactions between nanoclays and the non-polar polymer such as polypropylene (PP) in the nanoclay incorporated polymer composites are not satisfied. ${ }^{3}$ Many strategies have been utilized to improve the interfacial interactions for these composites. ${ }^{4}$ One of the convenient ways for the interfacial modification is the surface treatment of particles before their incorporation into the polymers. Among these methods, surface initiated grafting on the nanoparticles is effective for enhancing the interfacial interaction between the nanoparticles and the matrix through entanglement of the grafts attached to the nanoparticles with the matrix molecules. ${ }^{5}$ Alternatively, inclusion of the modifiers such as coupling agents, ${ }^{6,7}$ surfactants $^{8,9}$ and grafts ${ }^{10,11}$ etc. in the composites is also convenient and effective. The interaction mechanisms between the modifier and the nanoparticles including Van der Waals force, covalent bonding, hydrogen bonding, ionic bonding or physical entanglements have been reported. ${ }^{4}$

Halloysite nanotubes (HNTs), a type of tubular nanoclay with a similar geometry of carbon nanotubes, were recognized as the additive of polymers for better mechanical properties or thermal properties. ${ }^{12-14}$ Numerous surface modification methods were employed to improve the interfacial interactions between HNTs and the polymeric matrix. ${ }^{15}$ The interactions mechanism between the HNTs and the modifiers involved either covalent bonding or hydrogen bonding. Noticeably, HNTs are electron-deficient as the metal atoms of HNTs such as aluminum and ferrous are with capability to accept foreign electron on their empty orbital. ${ }^{16,17}$ Consequently, when the HNTs are intimately mixed with electron-rich species espe- cially the organics with conjugated structure, electron transferring from the organics to HNTs occurs. The special electron transferring interactions may be employed to increase the interfacial properties of the HNTs incorporated composites. Recently, we reported electron transferring interactions between HNTs and 2,5-bis(2-benzoxazolyl) thiophene (BBT) in $\mathrm{PP} / \mathrm{HNTs}$ nanocomposites. ${ }^{18}$ In the present work, 2,2'-(1,2ethenediyldi-4,1-phenylene) bisbenzoxazole (EPB), also commonly used as fluorescence brightener, was employed to investigate the effects of the interactions between HNTs and EPB on the morphology and the reinforcement of the PP/HNTs composites. The reinforcing mechanism of the EPB incorporated composites was correlated to the presence of the unique HNTs aggregates glued with EPB via electron transferring.

\section{EXPERIMENTAL}

\section{Raw Materials}

The isotactic PP, with a melt flow index of $2.84 \mathrm{~g} / 10 \mathrm{~min}$ (after ISO-1133: 1997(E)), was purchased from Lanzhou Petrochemical Co., China. The HNTs, mined from Yichang, Hubei, China, were purified according to reference. ${ }^{19}$ The elemental composition of HNTs by X-ray fluorescence (XRF) was determined as follows (wt.-\%): $\mathrm{SiO}_{2}, 58.91 ; \mathrm{Al}_{2} \mathrm{O}_{3}, 40.41$; $\mathrm{Fe}_{2} \mathrm{O}_{3}, 0.275 ; \mathrm{TiO}_{2}, 0.071$. 2,2'-(1,2-ethenediyldi-4,1-phenylene) bisbenzoxazole (EPB) with melting point of $319^{\circ} \mathrm{C}$ was industrial product and used as received. The chemical structure of the EPB was depicted as shown in Figure 1.

Characterization of Interaction between HNTs and EPB Surface Area and Pore Structure Analysis. The HNTs/EPB complex was prepared by blending HNTs and EPB vigorously. The Brunauer-Emmett-Teller (BET) surface area and pore structure of HNTs and the HNTs/EPB complex were investigated using nitrogen adsorption method with Micromeritics 


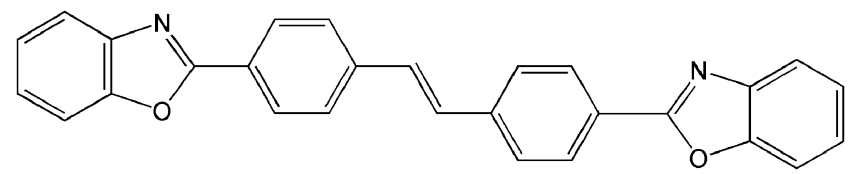

Figure 1. Chemical structure of EPB.

ASAP 2020. The pore-size distributions were computed by applying the Barrett-Joyner-Halenda (BJH) method.

In situ FT/IR. The experiment was performed with the MAGNA-IR760 (Nicolet Co, USA) equipped a heating furnace with the heating rate $5^{\circ} \mathrm{C} / \mathrm{min}$ from room temperature to $240^{\circ} \mathrm{C}$. The HNTs/EPB complex was pelleted with potassium bromide. Thirty-two consecutive scans were taken and their average was stored. Spectra were taken from 4000 to $400 \mathrm{~cm}^{-1}$. The resolution of the wavenumber is $4 \mathrm{~cm}^{-1}$.

\section{Preparation of PP/HNTs/EPB Composites}

A two-screw extruder was used to prepare the PP/HNTs/ EPB nanocomposites. The temperature setting of extruder from the hopper to the die was $180 / 190 / 195 / 200 / 200 / 190{ }^{\circ} \mathrm{C}$, and the screw speed was $100 \mathrm{rpm}$. The pelletized granules were dried for $5 \mathrm{~h}$ under $80^{\circ} \mathrm{C}$ and then injection molded under the temperature of $200{ }^{\circ} \mathrm{C}$. The weight ratio of PP and HNTs is constant of 100/30 and EPB content is variable in the range of $1-10$ phr relative to $\mathrm{PP}$.

\section{Characterization of the PP/HNTs/EPB Composites}

Scanning Electron Microscopy (SEM). The impact fractured surface of the composites was plated with a thin layer of gold before the observations. The SEM observations were done using a LEO1530 VP SEM machine with an attached X-ray energy dispersive spectrometer (X-EDS) was used to obtain elemental compositions of the composites.

Transmission Electron Microscopy (TEM). Ultrathin sections $(200 \mathrm{~nm})$ of the samples were cut using an unltramicrotome (EM ULTRACUT UC, Leica) and the sections were supported by holey carbon film on $\mathrm{Cu}$-grids. TEM analysis of the nanocomposites was carried out with Philips Tecnai 12 TEM. Mechanical Properties. Specimens for the tensile, flexural and impact testing were injection molded and measured according to ISO 527: 1993, ISO 178: 1993 and ISO 180: 1993 respectively. Zwick/Roell Z010, Instron 4465 and Zwick pendulum 5113.300 were used to perform the tensile, flexural and impact testing respectively.

Preparation of Precrack and Observation of Crack Growth. A notched composite bar was carefully cut with a razor to generate crack tip. Then, the precracking of the notched specimen was done with three-point flexural mode at a cross heading rate of $20 \mathrm{~mm} / \mathrm{min}$, which was illustrated as shown in Figure 2. The section containing the arrested crack tip was cut and coated with a thin layer of gold and observed using a Philips LEO1530 VP SEM machine at accelerating voltage of $20 \mathrm{kV}$ with an attached X-ray energy dispersive spectrometer (X-EDS) was used to obtain elemental compositions of the composites.

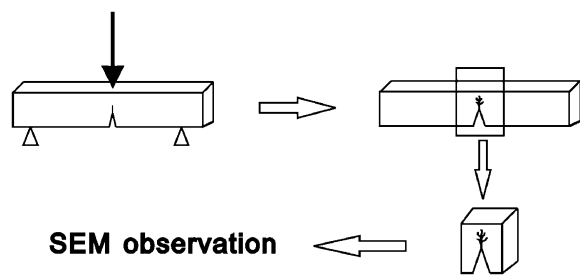

Figure 2. Scheme of the preparation of precrack and SEM sampling process at the crack tip.

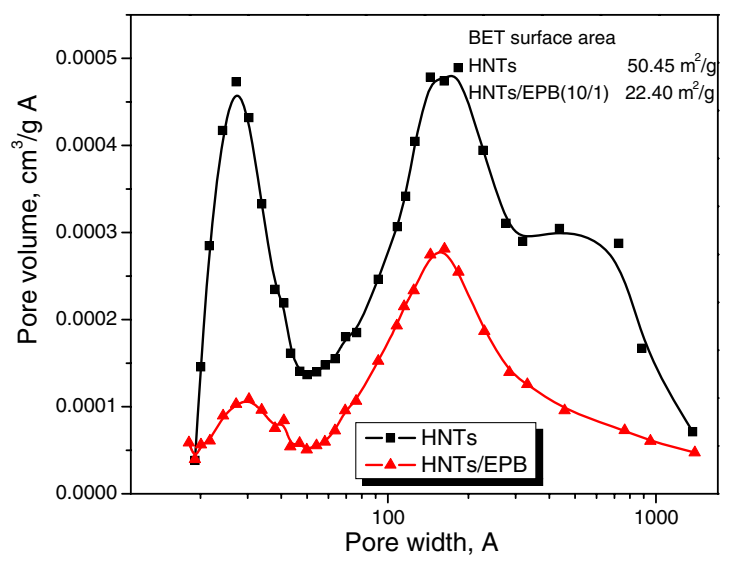

Figure 3. BJH pore-size distribution of HNTs and the HNTs/EPB complex.

\section{RESULTS AND DISCUSSION}

\section{Characterization of Interactions between HNTs and EPB}

The porous clay tend to absorb organics on their surface due to the interactions between clay and the organics such as electrostatic interaction, hydrogen bonding, ionic bonding and electron transferring interaction. ${ }^{20-23}$ The absorption of organics to clay can be verified by the porosity analysis method. Figure 3 shows the BJH pore size distribution of HNTs and the HNTs/EPB complex. It can be seen the pore volume of the HNTs/EPB is remarkably lower than that for HNTs, suggesting the absorption of EPB on the HNTs surface. The three peaks of the pore volume $v s$. pore width curve around 3, 17 and $50 \mathrm{~nm}$ are attributed to surface defects, the lumens of the nanotubes and pores among the tubes respectively. For the HNTs/EPB complex, the intensity of the peak around $3 \mathrm{~nm}$ characterizing the surface defects is largely decreased comparing with that of HNTs. In addition, the peak around $50 \mathrm{~nm}$ disappears, which can be attributed to occupation of the pores among the tubes by EPB. Therefore, it can be concluded that the EPB can be absorbed on the HNTs surface and filled the space among the HNTs. As expected, the BET surface area for HNTs/EPB complex is decreased from $50.5 \mathrm{~m}^{2} / \mathrm{g}$ for pristine HNTs to $22.4 \mathrm{~m}^{2} / \mathrm{g}$ for the complex. These results demonstrate that the EPB can strongly interact with HNTs and connect the nanotubes together.

In situ FT/IR technique is employed to investigate the interaction mechanisms between HNTs and EPB during 


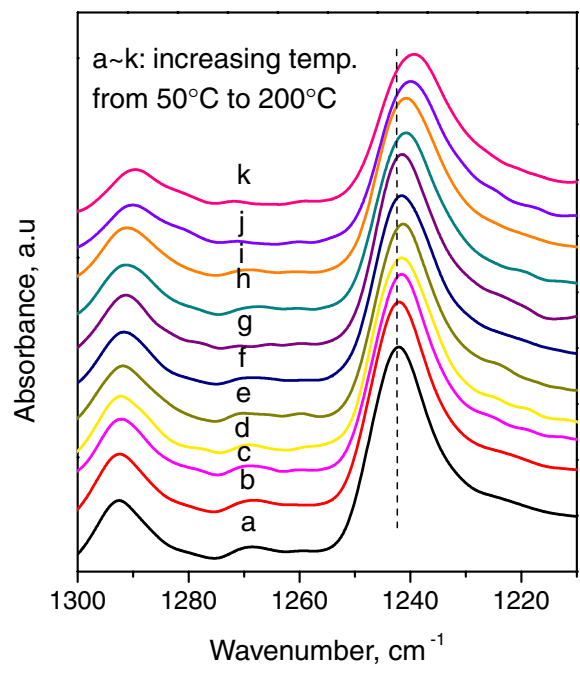

(a)

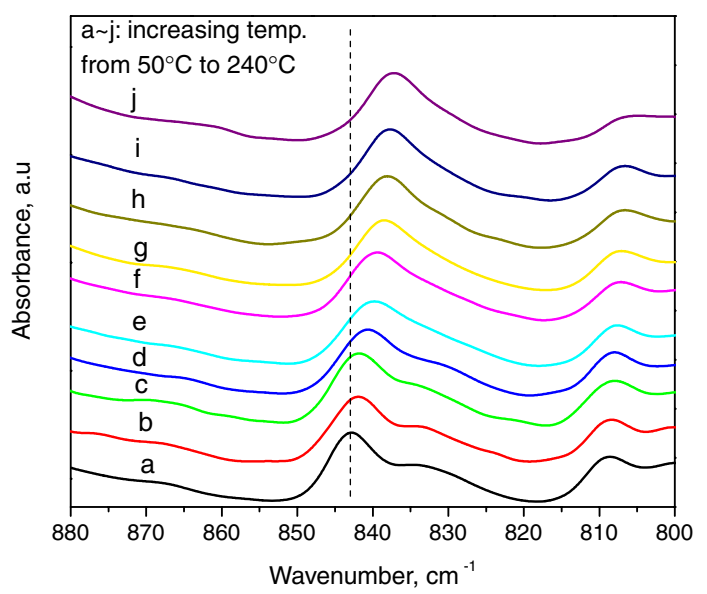

(b)

Figure 4. In situ FT/IR spectra of HNTs/EPB complex during heating: (a) $1300 \mathrm{~cm}^{-1}-1210 \mathrm{~cm}^{-1}$ region; (b) $880 \mathrm{~cm}^{-1}-800 \mathrm{~cm}^{-1}$ region.

heating. As the $\mathrm{N}$ and $\mathrm{O}$ atom in the benzoxazolyl group of EPB have lone electron pairs, EPB could donate electrons. Due to the unoccupied orbital of the metal atoms such as aluminum and ferrous in HNTs, HNTs could accept electrons. When the HNTs and EPB is intimately mixed, the electron transferring may take place. Figure 4 shows the evolution of the FT/IR spectrum of the HNTs/EPB complex during heating. With the evaluating temperature, the intensities of the absorption around $1240 \mathrm{~cm}^{-1}$, characterizing $=\mathrm{C}-\mathrm{O}-\mathrm{C}$ bond of benzoxazolyl group ${ }^{24}$ and $845 \mathrm{~cm}^{-1},{ }^{25}$ characterzing disubstituted benzene group of EPB, are decreased and blue-shifted consistently. These results can be explained by that the lone electron of $\mathrm{O}$ atoms on benoxazolyl group of EPB is transferred to HNTs upon heating. Highly conjugated structures of EPB make the electron transferring through the double bonds of benzene ring possible. Consequently, the FT/IR absorption of benzene ring also shows a decreased and blue-shifted trend. A similar phenomenon in HNTs/benzothiazole system was also observed. ${ }^{26}$ Figure 5 shows the photos of the HNTs/EPB mixture

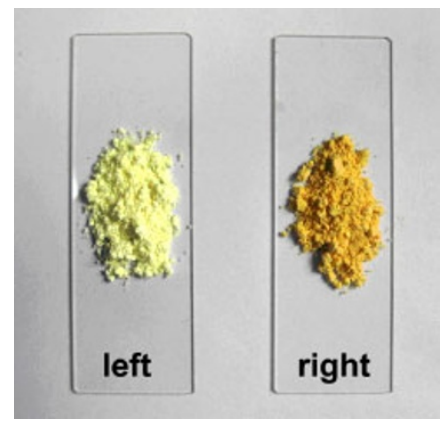

Figure 5. Photos of the HNTs/EPB mixture (left) and heat-treated HNTs/ EPB complex (right).

and the heat-treated HNTs/EPB complex. Before heat treatment the color of the HNTs/EPB mixture is light yellow, and it turn to dark yellow after the heat treatment. This also suggests the interactions between EPB and HNTs occur during heating. Color change is also an indication of electron transferring interaction. ${ }^{18,27}$ The electron transferring between HNTs and EPB is therefore confirmed.

\section{Morphology Observation of the PP/HNTs/EPB Compo- sites}

Figure 6 shows the SEM photos of the impact fractured surfaces of PP/HNTs/EPB composites. In absence of EPB, HNTs are uniformly dispersed in the matrix as the HNTs aggregates formed via hydrogen bonding can be easily separated during processing. Incorporating EPB to the composites brings some large HNTs aggregates in tens of micrometers. The unique HNTs aggregates are believed to be formed via the electron transferring interactions between HNTs and EPB during processing. EPB tends to be absorbed on HNTs and glues HNTs into aggregates. The EPB glued HNTs aggregates are much larger than the general HNTs aggregates formed via hydrogen bonding, which are occasionally observed in the PP/HNTs composite. Noticeably, as shown in the Figure 7, the morphology of PP/HNTs composites with higher EPB content is some different from that in the Figure 6. When more EPB is incorporated, apart from large spherical aggregates, rod-like aggregates in 2-5 micrometers are also observed. Figure $7 \mathrm{E}$ and $\mathrm{F}$ are the $\mathrm{X}$-ray energy dispersive spectra of the matrix and rod-like aggregates in the PP/HNTs/ EPB composites. It can be seen the main chemical composition of the matrix is carbon element, while the rod-like aggregates are composed with largely EPB and little of HNTs.

The aggregation of HNTs in presence of EPB is also observed by the TEM. Figure 8 shows the TEM photos of $\mathrm{PP} / \mathrm{HNT}$ /EPB composites. In absence of EPB, the HNTs are uniformly dispersed in PP matrix. Compared with other nanoclay such montmorillonite, the good dispersion of HNTs in the PP matrix is attributed to relatively lower interactions among the tubes and the tubular geometry of HNTs. ${ }^{18}$ Consistent with the SEM result, in presence of EPB, large HNTs aggregates in tens of micrometers are observed, indicating critical role of EPB in the formation of large HNTs aggregates. 

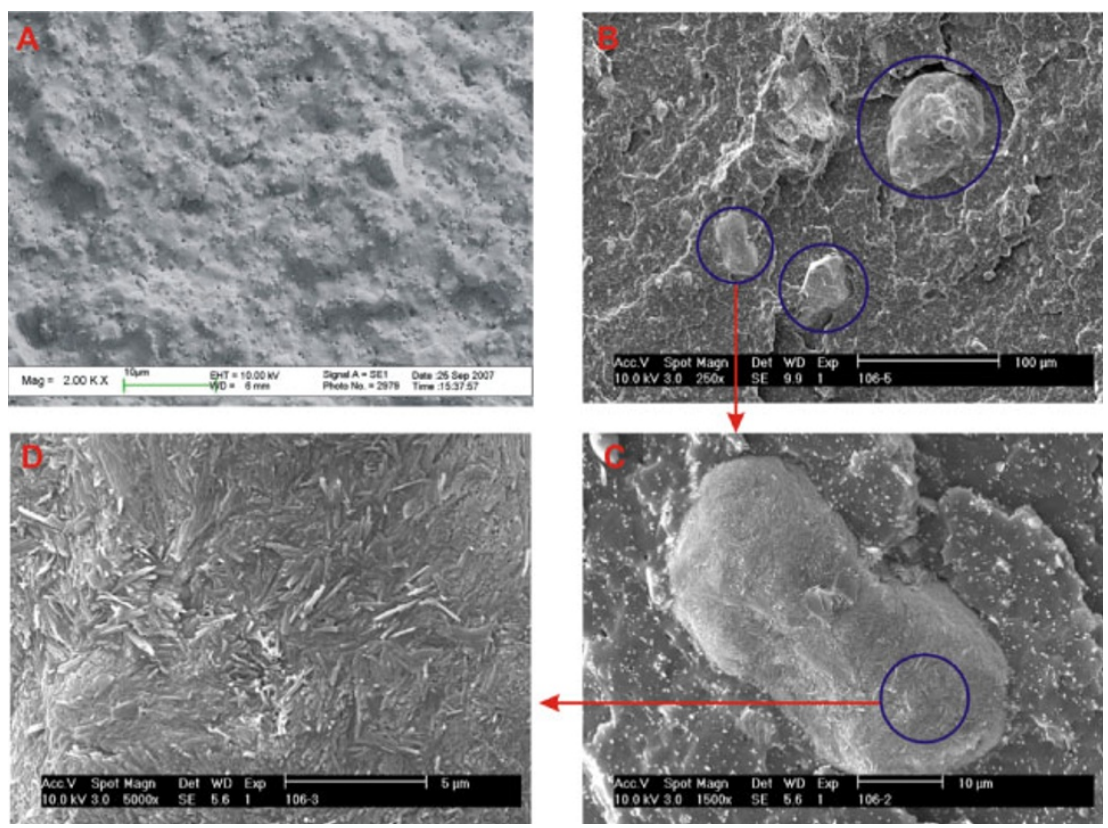

Figure 6. SEM photos of the impact fractured surfaces of the PP/HNTs/EPB composites: (A) PP/30 phr HNTs; (B)-(D) PP/30 phr HNTs/3 phr EPB.
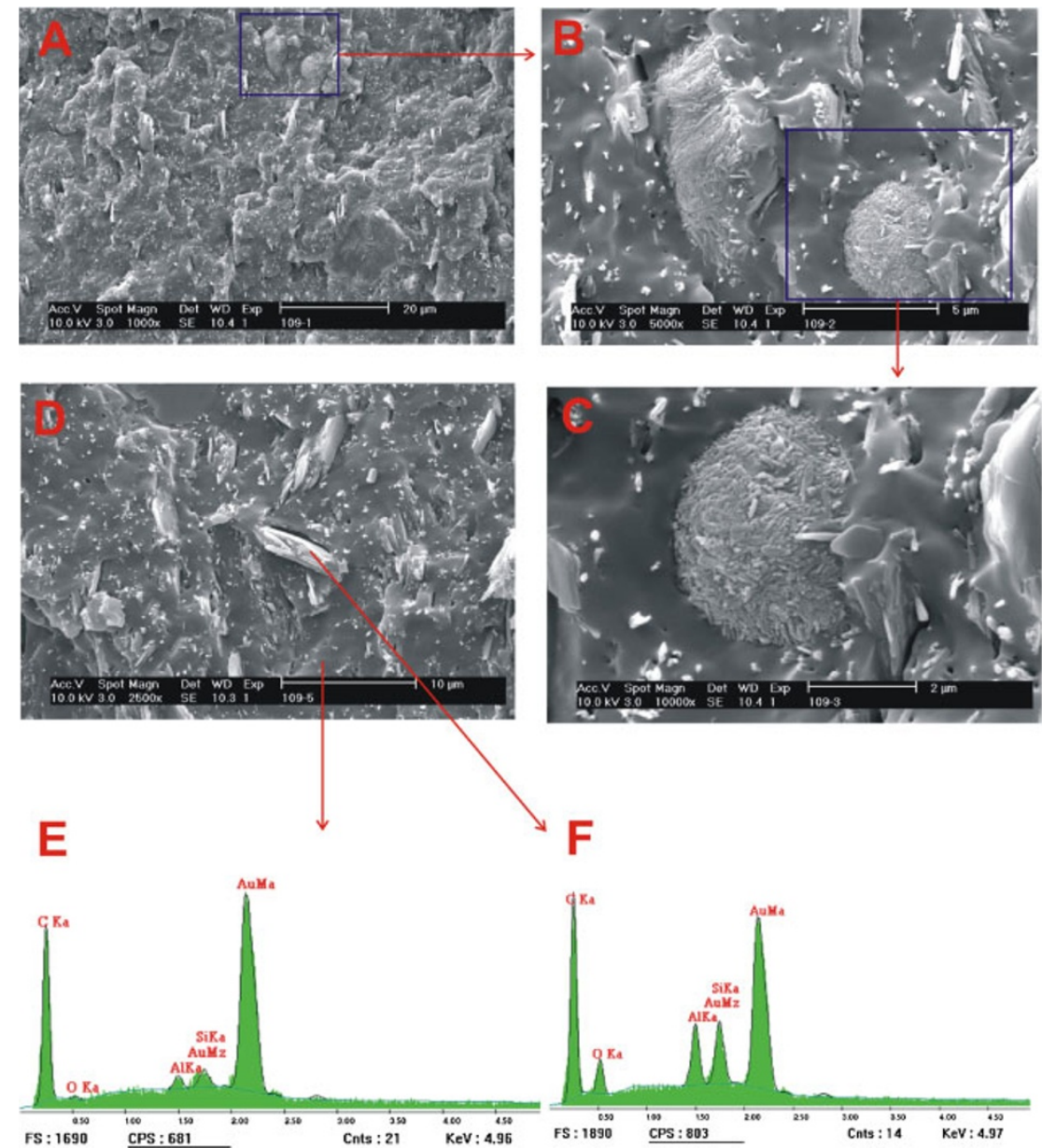

Figure 7. SEM photos of the impact fractured surfaces (A)-(D) and energy spectra analysis (E)-(F) of $P P / 30$ phr HNTs/10 phr EPB composites. 


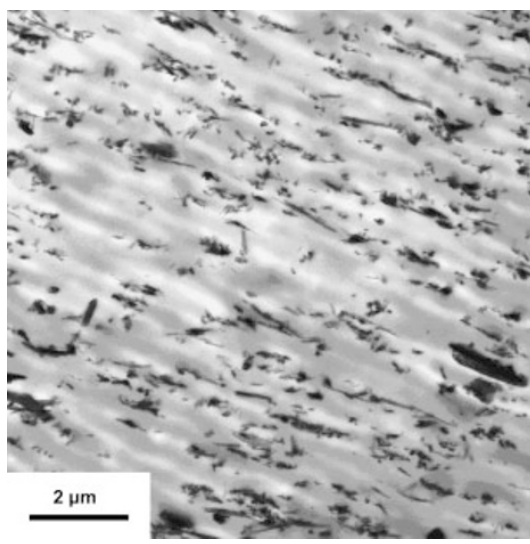

(a)

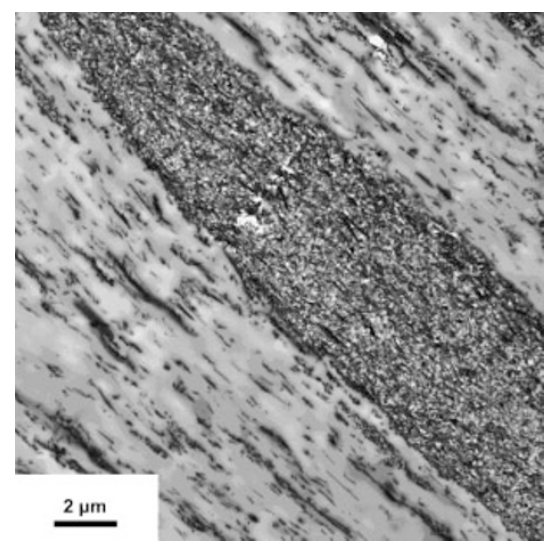

(b)

Figure 8. TEM photos of PP/HNTs/EPB composites: (a) PP/30 phr HNTs; (b) PP/30 phr HNTs/3 phr EPB.

Table I. Mechanical properties of PP/HNTs/EPB composites (Data in the parentheses indicates the standard deviations)

\begin{tabular}{ccccc}
\hline \multirow{2}{*}{ Samples } & $\begin{array}{c}\text { Tensile } \\
\text { Strength }\end{array}$ & $\begin{array}{c}\text { Flexural } \\
\text { Strength }\end{array}$ & $\begin{array}{c}\text { Flexural } \\
\text { Modulus }\end{array}$ & $\begin{array}{c}\text { Impact } \\
\text { Strength }\end{array}$ \\
\cline { 2 - 5 } & $\mathrm{MPa}$ & $\mathrm{MPa}$ & $\mathrm{GPa}$ & $\mathrm{kJ} / \mathrm{m}^{2}$ \\
\hline Neat PP & $32.1(0.5)$ & $35.2(1.3)$ & $1.21(0.04)$ & $5.36(0.47)$ \\
PP/HNTs $(100 / 30)$ & $31.8(0.4)$ & $46.2(1.0)$ & $1.78(0.07)$ & $3.85(0.15)$ \\
PP/EPB (100/3) & $33.3(0.2)$ & $38.9(1.0)$ & $1.38(0.11)$ & $6.78(0.05)$ \\
PP/EPB $(100 / 10)$ & $32.8(0.3)$ & $40.4(0.7)$ & $1.55(0.13)$ & $4.94(0.09)$ \\
PP/HNTs/EPB $(100 / 30 / 0.5)$ & $33.5(0.1)$ & $49.3(2.3)$ & $2.28(0.19)$ & $3.61(0.08)$ \\
PP/HNTs/EPB $(100 / 30 / 1)$ & $33.8(0.2)$ & $55.8(0.6)$ & $2.62(0.19)$ & $3.64(0.22)$ \\
PP/HNTs/EPB $(100 / 30 / 3)$ & $33.8(0.1)$ & $57.1(0.2)$ & $2.53(0.14)$ & $3.50(0.08)$ \\
PP/HNTs/EPB $(100 / 30 / 7)$ & $33.3(0.2)$ & $55.8(0.6)$ & $2.44(0.04)$ & $3.44(0.07)$ \\
PP/HNTs/EPB $(100 / 30 / 10)$ & $33.3(0.1)$ & $56.2(0.3)$ & $2.43(0.12)$ & $3.41(0.01)$ \\
\hline
\end{tabular}

\section{Mechanical Properties of the PP/HNTs/EPB Composites}

Mechanical properties of the composites were determined to examine the effects of interactions between HNTs and EPB and the unique morphology of the composites on the mechanical performances of PP/HNTs composites. The mechanical properties of the neat PP and the EPB incorporated PP/HNTs composites are summarized in Table I. The flexural strength and flexural modulus of the EPB included composites are substantially increased compared with those for the control sample. For example, incorporation of $3 \mathrm{phr}$ EPB brings $23.6 \%$ and $42.4 \%$ increments in flexural strength and flexural modulus respectively. The composites with higher EPB content (above $7 \mathrm{phr}$ ) show slightly decreased strength and modulus, which are correlated to the presence of the rod-like aggregates in the composites. The rod-like aggregates in the composites may act as the stress concentration centers and consequently lead to lowered modulus and strength. Although over loading EPB (above $7 \mathrm{phr}$ ) slightly deteriorated the mechanical properties, the mechanical properties of the PP/HNTs/EPB composites are still considerably higher than those of the control sample. All the EPB included PP/HNTs composites are suffered from slightly decreased impact strength. Noticeably, the composites containing EPB only (exclude HNTs), however, show little increases in mechanical properties compared with the neat PP.
Generally, the aggregation of particles is detriment for the reinforcing of composites, because the aggregates usually act as the stress-concentrated centers during the fracture process. Different from the general aggregates of inorganics particles, the formation of larger EPB glued HNTs aggregates permits entanglement of PP chains on the aggregates as the aggregation of HNTs in the presence of EPB leads to more disordered HNTs packing and consequently rough surface is generated. The extent of disorder is also crucial to the reinforcement of polymer composites ${ }^{28-31}$ and some aggregation process may leads to higher extent in disorder of particles. ${ }^{32,33}$ The entanglement of PP chains on the HNTs aggregates leads to slightly improved interfacial bonding, but it is not enough for affecting the overall mechanical performance. However, the entanglement of PP chains on the HNTs aggregates may change the fracture process and consequently change the mechanical properties. The changed fracture process and the reinforcing mechanism of the EPB included composite are illustrated below.

\section{Reinforcing Mechanism of the EPB Incorporated PP/HNTs Composites}

To investigate the reinforcing mechanism of the EPB incorporated PP/HNTs composites, the crack tip was arrested during the fracture process and the arrested crack tip was observed by the SEM. Figure 9 shows the arrested crack tip in the PP/HNTs/EPB composites. Upon loading, a macro-crack is observed around the position of the pre-crack. The cracks is branched and sharpened gradually and finally a clear crack tip is arrested. The enlarged crack propagation process near the crack tip is shown in Figure 9b. The white region represents the above mentioned HNTs aggregates. This is verified by the X-ray EDS results, which are shown in Table II. The elements $\mathrm{Ca}$ come from the impurities of HNTs. The main chemical composition of region $\mathrm{A}$ and $\mathrm{B}$ in Figure $9 \mathrm{c}$ is $\mathrm{Al}, \mathrm{Si}, \mathrm{O}$ and $\mathrm{C}$. As aluminum and silicon are the characteristic elements of HNTs, the HNTs aggregate in the composites consists of largely HNTs and little of EPB. It can be seen the crack can break and pass through the HNTs aggregate. After that, the 


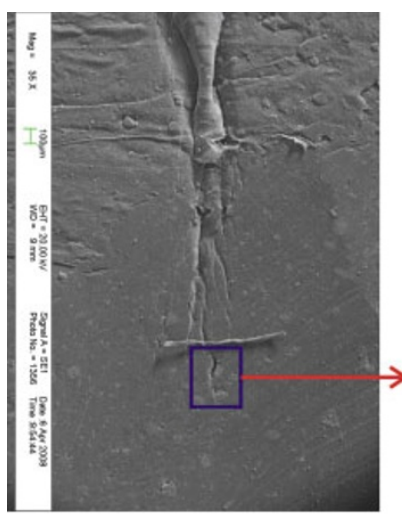

(a)

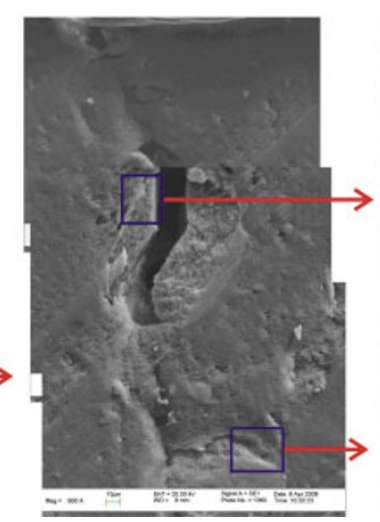

(b)

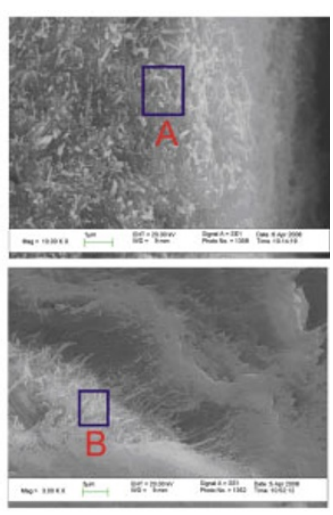

(c)

Figure 9. SEM photos of the arrested crack-tips for PP/30 phr HNTs/3 phr EPB composites.

Table II. X-Ray EDS Spectra data of the region in the Figure 7

\begin{tabular}{cccc}
\hline Region & Element & Weight percent (\%) & Atom percent (\%) \\
\hline \multirow{4}{*}{$\mathrm{C}$} & 4.94 & 8.37 \\
& $\mathrm{O}$ & 40.46 & 51.48 \\
& $\mathrm{Al}$ & 22.82 & 17.22 \\
& $\mathrm{Si}$ & 31.29 & 22.68 \\
& $\mathrm{Ca}$ & 0.49 & 0.25 \\
& Total & 100.00 & \\
& $\mathrm{C}$ & 9.50 & 14.95 \\
& $\mathrm{O}$ & 46.58 & 55.03 \\
& $\mathrm{Al}$ & 19.23 & 13.47 \\
$\mathrm{~B}$ & $\mathrm{Si}$ & 24.38 & 16.41 \\
& $\mathrm{Ca}$ & 0.30 & 0.14 \\
& Total & 100.00 & \\
\hline
\end{tabular}

crack propagation is continued and stopped finally by another HNTs aggregate. During the fracture process, the enhanced interfacial bonding in the EPB included composites allows the crack propagation in the HNTs aggregates. The crack propagation in the aggregates leads to the debonding of HNTs in the aggregates and consequently the fracture energy is dissipated. The crack propagation in the large HNTs aggregates absorbed extra fracture energy and consequently the strength and modulus are increased.

Noticeably, the presence of the HNTs aggregates is only beneficial to enhancing the flexural strength and modulus, the impact strength of the composites is decreased slightly compared with that for the control sample. This result can be explained by the following fact. The loading rate in impact test is much higher than that in flexural experiment $(2 \mathrm{~mm} / \mathrm{min})$. The enough slow loading rate permits the crack propagation through the HNTs aggregates as described above. When a high loading rate is applied, the crack propagation may be directed along the interface between the aggregate and the matrix. In this situation, the HNTs aggregates act as stress concentrated centers and consequently the impact strength of the composites is slightly deteriorated. Recently, Wu et al. suggested the toughening mechanism by HNTs aggregates in epoxy matrix. They suggested that the toughening mechanism was not suitable for the ductile polymers such as PE or PP, because crack tip would be blunted due to matrix shear yielding. ${ }^{34}$

The crack propagation process of the EPB incorporated PP/ HNTs composites are depicted in Scheme 1. Accordingly, the reinforcing mechanism responsible for the substantially improved mechanical properties of the EPB included composites are proposed as follow. Both dispersed HNTs and large EPB glued aggregates coexist in the EPB incorporated composites. When the composite is subjected to loading, the uniformly dispersed HNTs in the matrix can effectively transfer the stress. In addition, the microcracks are initiated around the materials defects and become the stress-concentrated centers. Upon further loading, these microcracks propagate into the aggregates. The cracks continue to propagate and extend into the matrix. Then they can be blunted by the deformation of the matrix or stopped by HNTs aggregate. This process may effectively absorb fracture energy. Therefore, both the presence of large HNTs aggregate and well dispersed HNTs are necessary in the present system for reinforcement of the PP composites.

\section{CONCLUSIONS}

The electron transferring interactions between HNTs and EPB was utilized to fabricate $\mathrm{PP} / \mathrm{HNT}$ s composite with improved mechanical properties. Upon heating the electron transferring between EPB and HNTs was taken place. Based on this mechanism, large HNTs aggregates in tens of micrometers were formed within the composites in presence of EPB during processing. The composites with EPB showed a substantially increased strength and modulus compared with those of the control sample. The reinforcement mechanism of EPB incorporated PP/HNTs composites were elucidated to the combination of the stress transferring by the well dispersed HNTs and the energy dissipation by cracking the HNTs aggregates during fracture process. The interaction between EPB and HNT played critical role in the formation of large HNTs aggregates and the propagation of crack into the aggregates during the fracture. The present work provides a novel reinforcing routine for 

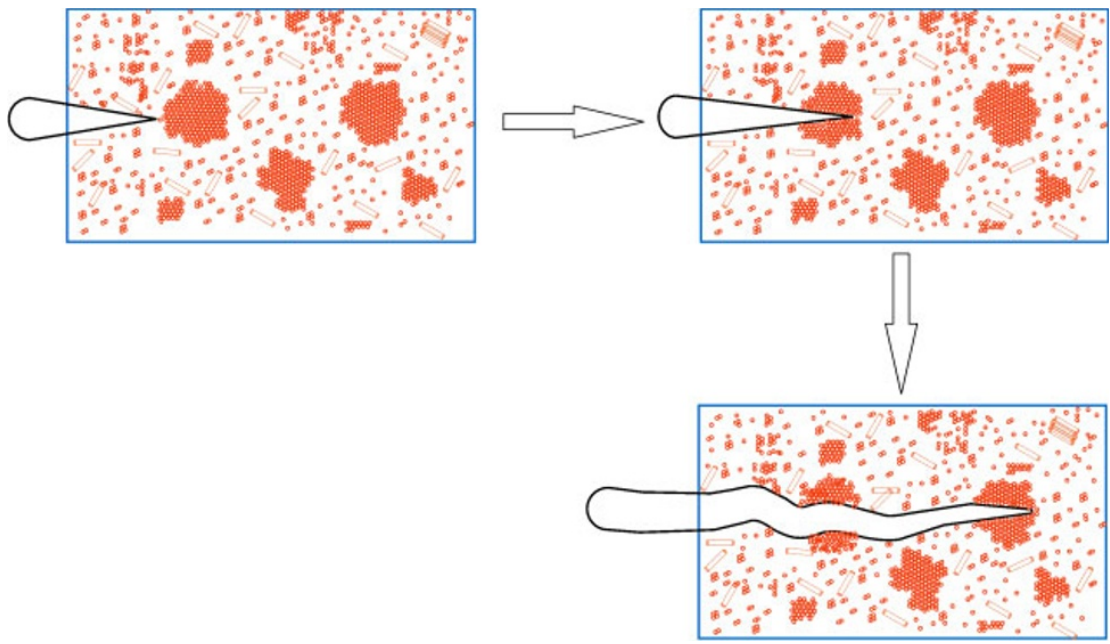

Scheme 1. Illustration of the crack initiation and propagation processes in the EPB included PP/HNTs composites as revealed by SEM photos.

nanoclay incorporated polymer composites via electron transferring mechanism.

Acknowledgment. We are grateful for the financial support by the National Natural Science Foundation of China with grant number of 50603005 and the Doctorate Foundation of South China University of Technology.

Received: May 31, 2008

Accepted: August 3, 2008 Published: September 18, 2008

\section{REFERENCES}

1. "Hybrid organic-inorganic composites. ACS Symposium Series 585," J. E. Mark, C. Y.-C. Lee, and P. A. Bianconi, Ed., American Chemical Society, Washington, DC, 1995.

2. E. Manias, A. Touny, L. Wu, K. Strawhecker, B. Lu, and T. C. Chung, Chem. Mater., 13, 3516 (2001).

3. A. Okada and A. Usuki, Macromol. Mater. Eng., 291, 1449 (2006).

4. B. Pukanszky and E. Fekete, Adv. Polym. Sci., 139, 109 (1999).

5. C. L. Wu, M. Q. Zhang, M. Z. Rong, and K. Friedrich, Compos. Sci. Technol., 65, 635 (2005).

6. N. N. Herrera, J. M. Letoffe, J. L. Putaux, L. David, and E. BourgeatLami, Langmuir 20, 1564 (2004).

7. A. Galanti, M. Laus, and M. Fiorini, Kautsch. Gummi Kunstst., 52, 21 (1999).

8. S. S. Ray and M. Okamoto, Prog. Polym. Sci., 28, 1539 (2003).

9. H. A. Stretz, D. R. Paul, R. Li, H. Keskkula, and P. E. Cassidy, Polymer, 46, 2621 (2005).

10. A. Usuki, N. Hasegawa, and M. Kato, Adv. Polym. Sci., 179, 135 (2005).

11. H. R. Fischer, L. H. Gielgens, and T. P. M. Koster, Acta Polym., 50, 122 (1999)

12. M. X. Liu, B. C. Guo, M. L. Du, X. J. Cai, and D. M. Jia, Nanotechnology 18, 455703 (2007).

13. M. L. Du, B. C. Guo, and D. M. Jia, Eur. Polym. J., 42, 1362 (2006).
14. M. L. Du, B. C. Guo, M. X. Liu, and D. M. Jia, Polym. Polym. Compos., 15, 321 (2007).

15. M. L. Du, Ph.D. Dissertation, South China University of Technology, 2007.

16. D. H. Solomon, B. C. Loft, and J. D. Swift, Clay Miner., 7, 389 (1968).

17. D. H. Solomon, Clay Clay Miner., 16, 31 (1968).

18. M. X. Liu, B. C. Guo, Q. L. Zou, M. L. Du, and D. M. Jia, Nanotechnology, 19, 205709 (2008).

19. D. G. Shchukin, G. B. Sukhorukov, R. R. Price, and Y. M. Lvov, Small, 1, 510 (2005).

20. M. A. Vicente, M. Sanchez-Camazano, M. J. Sanchez-Martin, M. D. Arco, C. Martin, V. Rives, and J. Vicente-Hernandez, Clays Clay Miner., 37, 157 (1989).

21. J. L. White and S. L. Hem, Ind. Eng. Chem. Prod. Res. Dev., 22, 665 (1983).

22. D. S. Warren, A. I. Clark, and R. Perry, Sci. Total Environ., 54, 157 (1986).

23. K. W. Weissmahr, S. B. Haderlein, R. P. Schwarzenbach, R. Hany, and R. Nuesch, Environ. Sci. Technol., 31, 240 (1997).

24. The Sadtler Handbook of Infrared Spectra. Bio-Rad Laboratories Inc., Informatics Division, 2004.

25. S. R. Sheng, M. Z. Cai, and C. S. Song, Acta Polym. Sin., 4, 490 (1999).

26. M. X. Liu, B. C. Guo, Y. D. Lei, M. L. Du, and D. M. Jia, J. Phys. Chem. B, submit.

27. D. L. Long, H. Abbas, P. Kogerler, and L. Cronin, Angew. Chem., Int Ed., 44, 3415 (2005)

28. D. Goritz, H. Raab, J. Frohlich, and P. G. Maier, Rubber Chem. Technol., 72, 929 (1999).

29. S. K. Friedlander, K. Ogawa, and M. Ullmann, J. Polym. Sci., Part B. Polym. Phys., 38, 2658 (2000).

30. M. Kluppel, Adv. Polym. Sci., 164, 1 (2003).

31. R. Kotsilkova, D. Fragiadakis, and P. Pissis, J. Polym. Sci., Part B: Polym. Phys., 43, 522 (2005).

32. Y. J. Suh, M. Ullmann, S. K. Friedlander, and K. Y. Park, J. Phys. Chem. B, 105, 11796 (2001).

33. M. L. Du, B. C. Guo, M. X. Liu, X. J. Cai, and D. M. Jia, J. Polym. Res., submit.

34. Y. P. Ye, H. B. Chen, J. S. Wu, and L. Ye, Polymer, 48, 6426 (2007). 